\title{
Antibacterial and antibiofilm activity of cinnamaldehyde against carbapenem-resistant Acinetobacter baumannii in Egypt: In vitro study
}

\author{
Sara H. Mohamed ${ }^{1}$, Dalia Salem²*, Magda Azmy ${ }^{3}$, Nevine S. Fam² \\ ${ }^{1}$ Department of Microbiology, National Organization for Drug Control and Research, Giza, Egypt. \\ ${ }^{2}$ Department of Microbiology, Theodor Bilharz Research Institute (TBRI), Giza, Egypt. \\ ${ }^{3}$ Department of Clinical Pathology and Electron Microscopy, Theodor Bilharz Research institute (TBRI), Giza, Egypt.
}

\begin{tabular}{l}
\hline ARTICLE INFO \\
\hline Received on: 25/09/2018 \\
Accepted on: 21/10/2018 \\
Available online: 30/11/2018 \\
\\
\hline Key words: \\
Acinetobacter baumannii, \\
biofilm, carbapenem- \\
resistant, cinnamaldehyde, \\
SEM.
\end{tabular}

ABSTRACT
Acinetobacter baumannii has emerged as a problematic nosocomial pathogen due to its antibiotic resistance as well
as its ability to colonize and cause serious infection among patients. This study aimed to evaluate the ability of $A$.
baumannii to form biofilms as well as to investigate the antibacterial activity of cinnamaldehyde against carbapenem-
resistant strains of $A$. baumannii. A total of 23 A. baumannii clinical strains were screened for their ability to form
a biofilm using tissue culture plate method. Cinnamaldehyde antibacterial ability was investigated on planktonic
cells and its biofilm inhibition ability was tested. Scanning electron microscopy (SEM) was applied to confirm the
antibiofilm effect of cinnamaldehyde. Biofilm formers ( $86.95 \%)$ were categorized into strong (17.39\%), moderate
(52.17\%), and weak (17.39\%). Cinnamaldehyde showed a strong antimicrobial activity against planktonic cells of $A$.
baumannii at low concentrations. The best antibiofilm activity was achieved at $1 / 2$ minimum inhibitory concentration
(MIC) and $1 / 4$ MIC causing inhibition percentages ranging from $49.5 \%$ to $71.2 \%$ and $18.5 \%$ to $29.6 \%$, respectively.
Cinnamaldehyde exerted strong antimicrobial and antibiofilm properties indicating their potential therapeutic value
that can be used as an option for treating biofilm associated clinical problems caused by $A$. baumannii.

\section{INTRODUCTION}

Acinetobacter baumannii (A. baumannii) is a wellknown pathogen which is responsible for hospital-acquired infections due to its remarkable ability to survive in the hospital environment as well as its rapidly acquire resistance determinants agents (Bogdan et al., 2017). Some of the difficulties in the treatment of the infections caused by this pathogen are its remarkable widespread resistance to different antibiotics and its ability to persist in nosocomial environments and medical devices (Abdulhasan et al., 2016).

One of the important mechanisms in the development of a multi-drug resistant (MDR) A. baumannii is the potential ability

\section{${ }^{*}$ Corresponding Author}

Dalia Salem, Department of Microbiology, Theodor Bilharz Research Institute (TBRI), Giza,Egypt.E-mail: drdaliasalem@gmail.com to form biofilms, which could explain its outstanding antibiotic resistance, survival properties, and owing protection from disinfectants and/or desiccation on the abiotic surfaces (Ivanković et al., 2017; Ryu et al., 2017). The adhesion ability of Acinetobacter strains is an important mechanism in its pathogenicity process, which is determined by specific and non-specific factors that vary among the strains (Imane et al., 2014).

Treatment options for MDR A. baumannii are extremely limited as a result for its resistant to most antimicrobial agents including carbapenems (Bae et al., 2016; Hu et al., 2017). Many natural compounds, such as plant extracts and secondary metabolites have been evaluated for antimicrobial activity (Nowotarska et al., 2017), and within this group, essential oils (EOs) are the most important members. In the recent years, many studies have done on a wide range of bacterial species showed the strong activity of EOs main components (Budzyńska et al., 2011; Kim et al., 2016; Mohamed et al., 2018a). Cinnamaldehyde, 
a major component of cinnamon EOs, occurs naturally in parts of cinnamon trees of the genus Cinnamomum. It has been proven to have antibacterial activities against a number of Gram-positive and Gram-negative bacteria (Jia et al., 2011; Khan et al., 2017). Therefore, our study was designed to evaluate the ability of $A$. baumannii to form biofilms as well as investigate the antimicrobial and antibiofilm activities of the cinnamaldehyde on clinical isolates of carbapenem-resistant $A$. baumannii.

\section{MATERIALS AND METHODS}

\section{Bacterial strains and antimicrobial susceptibility}

In this study, a total of 23 carbapenem-resistant $A$. baumannii clinical strains were collected from the different sources of Theodor Bilharz Research Institute (TBRI) during the period from November 2014 to December 2015 (Fam et al., 2017). All strains were previously identified by API 20E (bioMérieux, France) and confirmed using VITEK 2 compact system (bioMérieux, France). Antimicrobial susceptibility testing was done by using the disc diffusion method, and then minimum inhibitory concentration (MIC) was detected by VITEK2 compact system (bioMérieux, France).

\section{Quantitative biofilm production assay}

Colonies from 23 strains of fresh A. baumannii cultures (18 hours) were grown overnight at $37^{\circ} \mathrm{C}$ in brain heart infusion (BHI) broth medium for 24 hours. Biofilm formation was tested by adding $100 \mu \mathrm{l}$ of this standardized cell suspension to wells of microtiter plates that contained $100 \mu \mathrm{l}$ of fresh BHI media and incubating them at $37^{\circ} \mathrm{C}$ for 48 hours (Song et al., 2015). Thereafter, the medium was removed and planktonic cells were removed by washing the biofilms in phosphate buffered saline (PBS). After staining plates with 2\% crystal violet for $20 \mathrm{~m}$, excess stain was removed using water. The plates were air dried and then the dye was resolubilized with absolute ethanol. The optical density (OD) of each well was measured at 570 nm using Enzyme-Linked Immunosorbent Assay ELISA reader (Stat Fax 2100). Optical density cut-off value (ODc) was calculated using the equation: average $\mathrm{OD}$ of negative control $+(3 * \mathrm{SD}$ of negative control). Samples were considered positive when OD was $>0.228$.

\section{Antibacterial activity of cinnamaldehyde}

Nearly, $100 \mu \mathrm{l}(0.5 \mathrm{McFarland})$ of the bacterial culture was inoculated into each well of a 96-well microtiter plate containing $100 \mu \mathrm{l}$ trans-cinnamaldehyde (Sigma Aldrich, St. Louis, MO) at different concentrations $(0.027-28 \mathrm{mg} / \mathrm{ml})$. Wells without cinnamaldehyde were used as a positive control while those without bacteria were considered as negative controls. After 24 hours incubation at $37^{\circ} \mathrm{C}$, the wells were visually inspected for the growth. The MIC was considered as the lowest concentration of cinnamaldehyde that inhibits the bacterial growth. Nearly, 100 $\mu 1$ of culture from each clear well in the MIC assay was placed on a Mueller-Hinton agar plate and incubated at $37^{\circ} \mathrm{C}$ for 24 hours. The lowest concentration of cinnamaldehyde that inhibits the bacterial growth on an agar plate was considered as the minimum bactericidal concentration (MBC) (Jia et al., 2011).

\section{Antibiofilm activity of cinnamaldehyde}

This test was performed on four strains that showed strong biofilm formation ability in the biofilm production assay.
The effect of different concentrations of cinnamaldehyde to inhibit the ability of $A$. baumannii cells to form a biofilm was assessed using the TCP method adopted by Adukwu et al. (2012) with minor modifications. Nearly, $100 \mu \mathrm{l}$ of $0.5 \mathrm{McF}$ arland bacterial culture was dispensed into each well of 96-well polystyrene microtiter plates in the presence of $100 \mu \mathrm{l}$ of cinnamaldehyde different concentrations, and plates were incubated at $37^{\circ} \mathrm{C}$ for 48 hours. Antimicrobial agent free wells served as positive controls for the biofilm growth. After incubation, the medium and nonadherent cells were removed and wells were washed three times with sterile PBS. The plates were air dried and then the dye was resolubilized with absolute ethanol. The OD of each well was measured at $570 \mathrm{~nm}$ using ELISA reader (Stat Fax 2100). Each assay was performed in triplicates.

\section{Scanning electron microscopy (SEM)}

SEM was used to investigate the effect of cinnamaldehyde on Acinetobacter biofilm. Sections of polystyrene tubes that coated with bacterial biofilm were processed similarly with the method described by Mohamed et al. (2018a). Then examined with Philips XL30 scanning electron microscope (Eindhoven, Netherlands) operated at $20 \mathrm{kV}$.

\section{Statistical analysis}

Quantitative variables were expressed as mean \pm standard deviation (SD), and data were analyzed by SPSS statistical software, version 12.0. One-way analysis of variance and a post hoc multiple comparisons (Duncan test) were used to compare the effect of different concentrations of cinnamaldehyde on Acinetobacter strains. Statistically, the values were considered significant at $p<0.05$.

\section{RESULTS}

All strains were found to be resistant to carbapenems when tested by E-test strips including doripenem showing MIC $>32 \mu \mathrm{g} / \mathrm{ml}$. Most of the strains also showed resistance to multiple classes of antibiotics including; $\beta$-lactams, aminoglycosides, quinolones, and trimethoprim/sulphamethoxazole (Fam et al., 2017).

In this study, biofilm formation assay was performed for 23 A. baumannii MDR strains, and the biofilm formation ability was determined by the estimation of OD570 values. Out of 23 Acinetobacter strains, $20(86.95 \%)$ strains were able to form biofilms (Table 1). The formation ability of biofilm was categorized as follows: 4 isolates as strong formers (17.39\%), 12 isolates as moderate formers $(52.17 \%), 4$ isolates as weak formers $(17.39 \%)$, and 3 isolates as non-biofilm formers (13.04\%).

The four strong biofilm formers were chosen to test the antimicrobial and antibiofilm activity of Cinnamaldehyde. Our results showed a strong antimicrobial activity against planktonic cells of $A$. baumannii, at which MIC and MBC were detected at 0.875 and $1.75 \mathrm{mg} / \mathrm{ml}$, respectively, for the four strains.

The antibiofilm activity of cinnamaldehyde was measured at different sub-inhibitory concentrations ( $1 / 2 \mathrm{MIC}, 1 / 4$ MIC, $1 / 8 \mathrm{MIC}$, and $1 / 16 \mathrm{MIC}$ ). The best antibiofilm activity was achieved at $1 / 2$ MIC which caused inhibition percentages ranging from $49.452 \%$ to $71.158 \%$. Following $1 / 2$ MIC concentration, $1 / 4$ MIC, $1 / 8$ MIC, and $1 / 1616$ MIC caused inhibition percentages ranging from $18.540 \%$ to $29.606 \%, 11.749 \%$ to $13.337 \%$, and $5.841 \%$ to $12.120 \%$, respectively. 
Table 1. Screening of biofilm formation ability among Acinetobacter baumannii strains at $570 \mathrm{~nm}$.

\begin{tabular}{|c|c|c|c|c|c|}
\hline \multirow{2}{*}{ Strains } & \multicolumn{3}{|c|}{ OD570 } & \multirow{2}{*}{ Average \pm SD } & \multirow{2}{*}{ Biofilm formation ability } \\
\hline & Ex. 1 & Ex. 2 & Ex. 3 & & \\
\hline R2 & 0.297 & 0.435 & 0.649 & $0.460 \pm 0.177$ & Weak biofilm \\
\hline R7 & 0.219 & 0.245 & 0.214 & $0.226 \pm 0.016$ & No biofilm \\
\hline R8 & 0.78 & 0.71 & 0.724 & $0.738 \pm 0.037$ & Moderate biofilm \\
\hline R9 & 1.66 & 1.378 & 1.433 & $1.490 \pm 0.149$ & Strong biofilm \\
\hline R11 & 0.804 & 0.804 & 0.899 & $0.835 \pm 0.054$ & Moderate biofilm \\
\hline R13 & 0.322 & 0.315 & 0.57 & $0.402 \pm 0.145$ & Weak biofilm \\
\hline R14 & 1.258 & 1.706 & 1.602 & $1.522 \pm 0.234$ & Strong biofilm \\
\hline R18 & 0.279 & 0.389 & 0.383 & $0.350 \pm 0.061$ & Weak biofilm \\
\hline R24 & 0.716 & 0.562 & 0.464 & $0.581 \pm 0.127$ & Moderate biofilm \\
\hline $\mathbf{R} 28$ & 0.53 & 0.524 & 0.391 & $0.482 \pm 0.078$ & Moderate biofilm \\
\hline R31 & 0.6 & 0.84 & 0.331 & $0.590 \pm 0.254$ & Moderate biofilm \\
\hline R34 & 2.103 & 2.366 & 2.103 & $2.191 \pm 0.151$ & Strong biofilm \\
\hline R36 & 1.273 & 1.095 & 1.466 & $1.278 \pm 0.185$ & Strong biofilm \\
\hline R41 & 0.531 & 0.601 & 0.578 & $0.57 \pm 0.0356$ & Moderate biofilm \\
\hline R45 & 0.689 & 0.677 & 0.747 & $0.704 \pm 0.037$ & Moderate biofilm \\
\hline R51 & 0.703 & 0.493 & 0.621 & $0.606 \pm 0.105$ & Moderate biofilm \\
\hline R58 & 0.217 & 0.252 & 0.21 & $0.226 \pm 0.022$ & No biofilm \\
\hline R71 & 0.796 & 0.743 & 0.697 & $0.745 \pm 0.049$ & Moderate biofilm \\
\hline R73 & 0.758 & 0.684 & 0.467 & $0.636 \pm 0.151$ & Moderate biofilm \\
\hline R74 & 0.796 & 0.897 & 0.824 & $0.839 \pm 0.052$ & Moderate biofilm \\
\hline R76 & 0.651 & 0.725 & 0.752 & $0.709 \pm 0.052$ & Moderate biofilm \\
\hline R77 & 0.344 & 0.34 & 0.539 & $0.408 \pm 0.113$ & Weak biofilm \\
\hline R80 & 0.207 & 0.229 & 0.228 & $0.221 \pm 0.012$ & No biofilm \\
\hline C & 0.202 & 0.204 & 0.215 & $0.207 \pm 0.007$ & - \\
\hline
\end{tabular}

$\mathrm{OD}=$ optical density $\mathrm{SD}=$ standard deviation; $\mathrm{C}=$ control.

Sub-inhibitory concentrations of cinnamaldehyde ( $1 / 2$ MIC and $1 / 4$ MIC) were found to cause inhibition percentage, which is not significantly different when $1 / 4$ MIC was used but was significantly different for strain R36 when $1 / 2$ MIC was used among different Acinetobacter strains (Fig. 1).

SEM was employed to confirm the effect of cinnamaldehyde on Acinetobacter biofilm at the concentrations of $1 / 2$ MIC and $1 / 4$ MIC (Fig. 2). Colonized cells were detected in the untreated biofilm (control) (Fig. 2A and a), while for biofilm treated with $1 / 4 \mathrm{MIC}$ cinnamaldehyde concentration, lower number of colonized cells was shown (Fig. 2B and b). On the other hand, noticeable inhibition of $A$. baumannii biofilm formation was detected in $1 / 2$ MIC cinnamaldehyde concentration treated biofilm (Fig. 2C and c) which confirmed the results obtained phenotypically.

\section{DISCUSSION}

Nowadays, A. baumannii has emerged as a pathogen that related to outbreaks of colonization and infection of critically ill patients (Imane et al., 2014). Resistant strains of A. baumannii are known to be one of the most difficult treating (Almaghrabi et al., 2018; Ramanathan et al., 2017). The ability of resistant $A$. baumannii strains to spread widely seems to depend on some virulence factors that allow bacterial colonization such as biofilm formation ability (Reena et al., 2017), which plays an effective role in medical device-associated infections (Longo et al., 2014).
In our study, $86.95 \%$ of $A$. baumannii strains were found to have the ability to form a biofilm, which considered as a high percentage when compared with other recent studies reporting 70\% in Brazil (de Campos et al., 2016), 72.91\% in Iran (Dehbalaei et al., 2017), 76.92\% in India (Anish et al., 2017), $85.6 \%$ in Lebanon (Dahdouh et al., 2016), but lower than that was reported in Iraq (91.25\%) (Authman et al., 2017). While in our country Egypt, several studies reported the biofilm formation among $A$. baumannii by the percentages of $60 \%, 62.5 \%$, and 84\% (Elhabibi and Ramzy, 2017; El-Kady, 2015; Mansour and Rhman, 2012). This high percentage of biofilm formation among our isolates is a serious concern. It is known that bacterial cells acquire more resistance to multiple drugs within the biofilm mode than the planktonic one. Thus, function the biofilm formed by $A$. baumannii encompasses its ability to resist antimicrobial therapies as well as to protect bacterial cells from external stresses such as dehydration and limited availability of nutrients (Badave and Dhananjay, 2015).

Another serious concern about $A$. baumannii infections is the detection of an increasing rate of resistance to multiple antibiotics and in some cases, resistant to all available antibiotics (Bialvaei et al., 2017). The wide spread antibiotic resistance observed is now posing a serious public health concern and warning of a return to the pre-antibiotic era. Recently, an interest was given to natural compounds which have antimicrobial and anti-adherence activities (Gupta and Birdi, 2017; Mohamed et al., 2018b). 


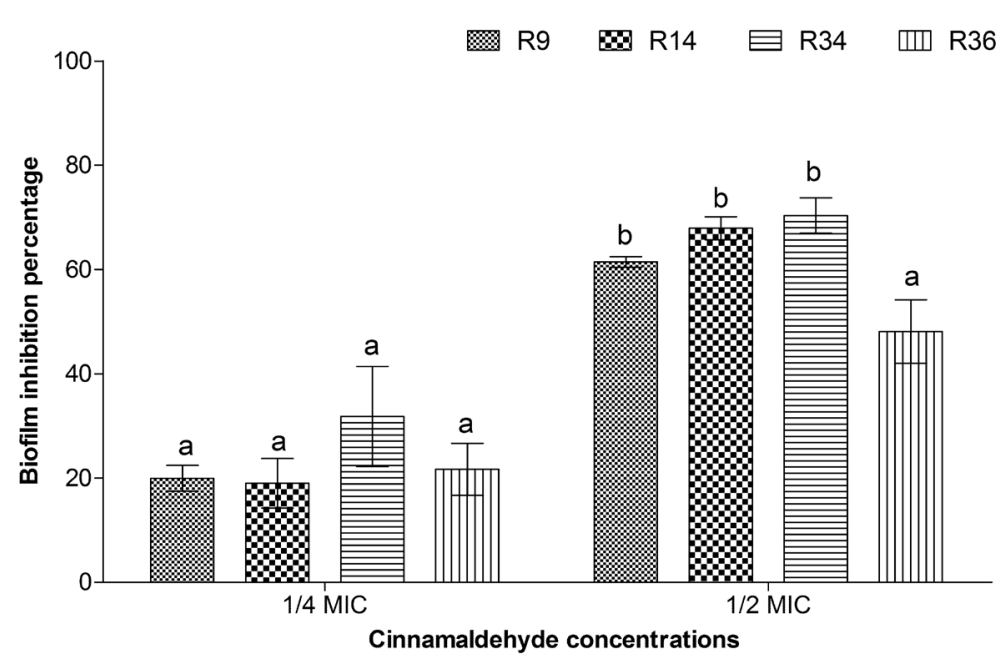

Figure 1. Biofilm inhibition percentage caused by the best cinnamaldehyde concentrations ( $1 / 2 \mathrm{MIC}$ and $1 / 4 \mathrm{MIC})$ against the four strong biofilm formers strains of Acinetobacter.

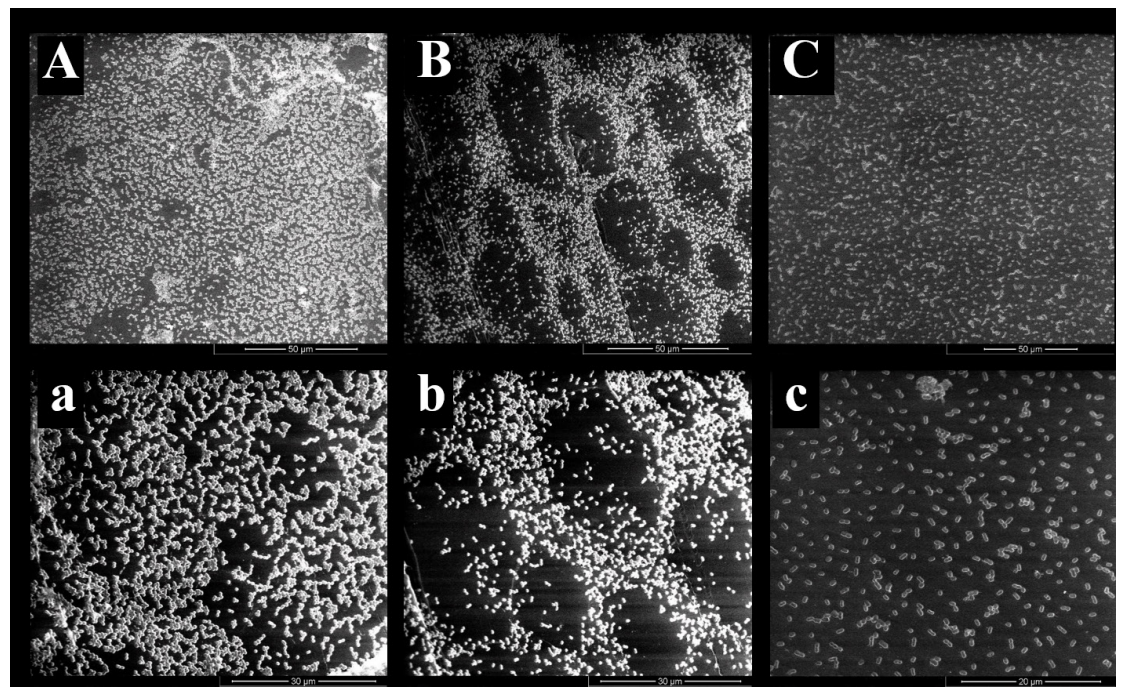

Figure 2. SEM visualization of $A$. baumannii biofilms. (A and a) Untreated biofilms at magnification 1,850× and 4,000×, (B and b) biofilms treated with $1 / 4$ MIC of cinnamaldehyde at magnification $2,000 \times$ and 4,000×, and $(\mathrm{C}$ and $\mathrm{c}$ ) biofilms treated with $1 / 2 \mathrm{MIC}$ cinnamaldehyde at magnification $1,600 \times$ and $6,000 \times$.

Mansour and Rahman (2012) indicated the importance of controlled antibiotic usage and appliance of proper hospital infection control measures. While recently Bogdan et al. (2017) stated that there are no published extensive studies about the effect of sub MICs of antimicrobials on the virulence factors of $A$. baumannii, to face the growing global problem of $A$. baumannii multi resistance to antimicrobial drugs.

Our study showed a strong antibacterial and antibiofilm activity at low concentrations of cinnamaldehyde against strong biofilm producing strains of $A$. baumannii clinical strains at which MIC and MBC were detected at 0.875 and $1.75 \mathrm{mg} / \mathrm{ml}$, respectively. Anish et al. (2017) tried to use chemical compounds in the war against biofilm production and antimicrobial resistance of certain bacteria, stating that $55 \%-75 \%$ reduction of biofilm was seen by using Ethylene Diamine Tetraacetic Acid EDTA. Other studies tried to use natural compounds such as Cinnamaldehyde against different bacterial species, concluded that Cinnamaldehyde represent potential antimicrobial agents for treating wound infections and suggesting that it may represent an alternative strategy to control bacterial infections as it presents the ability to inhibit bacterial growth without promoting an adaptive phenotype (Ferro et al., 2016; Jia et al., 2011).

This antimicrobial activity of cinnamaldehyde was studied recently and mechanisms were suggested such as (a) interacting with the cell membrane rapid inhibition of energy metabolism (Friedman, 2017); (b) its hydrophobicity enables it to enter and disturb the lipid bilayer of the cell membrane and cause increased permeability to protons and the exit of critical molecules and ions from bacterial cells ultimately leading to bacterial cell death (Jia et al., 2011); and (c) by affecting membrane integrity, energy generation, and extended-spectrum beta-lactamase protein dependent drug resistance (Thakre et al., 2016).

Concerning its antibiofilm activity, extensive research exploring alternative strategies for microbial biofilm control 
has highlighted the efficacy of plant-derived antimicrobials in controlling biofilm formation of human pathogens. Extracellular polymeric substances (EPS) in biofilms are responsible for binding cells and other particulate materials to each other's and to the surface, as well as for the development of biofilm structure and maturation (Azizun et al., 2013; Thummeepak et al., 2016). Cinnamaldehyde, at a certain concentration significantly reduces quorum sensing (QS) signaling, which results in reduction or prevention of EPS formation, and this may explain its strong antibiofilm activity (Niu et al., 2006; Bai A and Vittal, 2014). Our results strongly support such findings as both tissue culture plate method and scanning electron microscope studies showed a marked inhibition of $A$. baumannii biofilm formation in $1 / 2 \mathrm{MIC}$ cinnamaldehyde concentration treated biofilms.

\section{CONCLUSION}

Cinnamaldehyde exerts strong antimicrobial and antibiofilm activities against carbapenem-resistant $A$. baumannii strains which can be used as an option for treating biofilm associated clinical problems caused by this pathogen.

\section{CONFLICT OF INTEREST}

Authors declare that there is no conflict of interest exists.

\section{FUNDING}

This work was supported by Theodor Bilharz Research Institute (TBRI) as a part of an ongoing internal project No. 96 D.

\section{REFERENCES}

Abdulhasan GA, Alzubaidy SK, Abed IJ. Effect of sub-inhibitory and inhibitory concentrations of some antibiotics and rosemary essential oil (Rosmarinus officinalis L.) on biofilm formation of Klebsiella pneumoniae. World J Exp Biosci, 2016; 4:130-5.

Adukwu EC, Allen SCH, Phillips CA. The anti-biofilm activity of lemongrass (Cymbopogon flexuosus) and grapefruit (Citrus paradisi) essential oils against five strains of Staphylococcus aureus. J Appl Microbiol, 2012; 113:1217-27.

Almaghrabi MK, Joseph MRP, Assiry MM, Hamid ME. Multidrug-resistant Acinetobacter baumannii: an emerging health threat in Aseer Region, Kingdom of Saudi Arabia. Can J Infect Dis Med Microbiol, 2018; 2018:9182747; doi:10.1155/2018/9182747

Anish C, Abhisek R, Radha M. Evaluation of biofilm production in Acinetobacter baumanii with reference to imipenem resistance. Inter J Sci Res Pub, 2017; 7:732-7.

Authman SH, Ali FS, Marjani MFA. Biofilm formation in imipenem-resistant Acinetobacter baumannii from the intensive care unit. J Glob Pharma Technol, 2017; 10:404-11.

Azizun N, Shaheda A, Miah MRA. Association of biofilm formation with antimicrobial resistance among the Acinetobacter species in a tertiary care hospital in Bangladesh. J Med, 2013; 14:28-32.

Badave GK, Dhananjay K. Biofilm producing multidrug resistant Acinetobacter baumannii: an emerging challenge. J Clin Diagnostic Res, 2015; 9:DC08-10.

Bae S, Kim MC, Park SJ,Kim HS, Sung H, Kim MN, Kim SH, Lee SO, Choi SH, Woo JH, Kim YS, Chong YP. In vitro synergistic activity of antimicrobial agents in combination against clinical isolates of colistinresistant Acinetobacter baumannii. Antimicrob Agents Chemother, 2016; 60:6774-9.

Bai AJ, Vittal RR. Quorum sensing inhibitory and anti-Biofilm activity of essential oils and their in vivo efficacy in food systems. Food Biotechnol, 2014; 28:269-92.

Bialvaei AZ, Kouhsari E, Salehi-Abargouei A, Amirmozafari
N, Ramazanzadeh R, Ghadimi-Daresajini A, Sedighi M. Epidemiology of multidrug-resistant Acinetobacter baumannii strains in Iran: a systematic review and meta-analysis. J Chemother, 2017; 29:327-37.

Bogdan M, Drenjancevic D, Harsanji Drenjancevic I, Bedenic B, Zujic Atalic V, Talapko J, Vukovic D. In vitro effect of subminimal inhibitory concentrations of antibiotics on the biofilm formation ability of Acinetobacter baumannii clinical isolates. J Chemother, 2017; 9478:1-9.

Budzyńska A, Wieckowska-Szakiel M, Sadowska B, Kalemba D, Rózalska B. Antibiofilm activity of selected plant essential oils and their major components. Pol J Microbiol, 2011; 60:35-41.

De Campos PA, Royer S, da Fonseca Batistão DW, Araújo BF, Queiroz LL, de Brito CS, Gontijo-Filho PP, Ribas RM. Multidrug resistance related to biofilm formation in Acinetobacter baumannii and Klebsiella pneumoniae clinical strains from different pulsotypes. Curr Microbiol, 2016; 72:617-27.

Dahdouh E, Hajjar M, Suarez M, Daoud Z. Acinetobacter baumannii isolated from Lebanese patients: phenotypes and genotypes of resistance, clonality, and determinants of pathogenicity. Front Cell Infect Microbiol, 2016; 6:1-10.

Dehbalaei MA, Najar-Peerayeh S, Taherikalani M, Behmanesh M. Clinical isolates of Acinetobacter baumannii from tehran hospitals: pulsed-field gel electrophoresis characterization, clonal lineages, antibiotic susceptibility, and biofilm-forming ability. Jundishapur J Microbiol, 2017; 10:e13790; doi:10.5812/jjm.13790

El-Kady RA. Acinetobacter baumannii : correlation between biofilm production and multidrug resistance. Int J Adv Res, 2015; 3:691-9.

Elhabibi T, Ramzy S. Biofilm production by multi drug resistant bacterial pathogens isolated from patients in intensive care units in Egyptian hospitals. J Microb Biochem Technol, 2017; 09:151-8.

Fam N, Gamal D, Azmy M, Gamal D. Antimicrobial efficacy of doripenem colistin combination on carbapenem-resistant Acinetobacter baumanii isolates by E-test agar dilution and ultrastrastructural methods. Egypt J Med Microbiol, 2017; 26:1-7.

Ferro TA, Araújo JM, Dos Santos Pinto BL, Dos Santos JS, Souza EB, da Silva BL, Colares VLP, Novais TMG, Filho CMB, Struve C, Calixto JB, Monteiro-Neto V, da Silva LC, Fernandes ES. Cinnamaldehyde inhibits Staphylococcus aureus virulence factors and protects against infection in a Galleria mellonella model. Front Microbiol, 2016; 7:1-10.

Friedman M. Chemistry, antimicrobial mechanisms, and antibiotic activities of cinnamaldehyde against pathogenic bacteria in animal feeds and human foods. J Agric Food Chem, 2017; 65:10406-23.

Gupta PD, Birdi TJ. Development of botanicals to combat antibiotic resistance. J Ayurveda Integr Med, 2017; 8:266-75.

Hu YF, Hou CJY, Kuo CF, Wang NY, Wu AY, Leung CH, Liu CP, Yeh HI. Emergence of carbapenem-resistant Acinetobacter baumannii ST787 in clinical isolates from blood in a tertiary teaching hospital in Northern Taiwan. J Microbiol Immunol Infect, 2017; 50:640-5.

Imane M, Hafida H, Samia B, Ryad D. Biofilm formation by Acinetobacter baumannii isolated from medical devices at the intensive care unit of the University Hospital of Tlemcen (Algeria). Afr J Microbiol Res, 2014; 8:270-6.

Ivanković T, Goić-Barišić I, Hrenović J. Reduced susceptibility to disinfectants of Acinetobacter baumannii biofilms on glass and ceramic. Arh Hig Rada Toksikol, 2017; 68:53-8.

Jia P, Xue YJ, Duan XJ, Shao SH. Effect of cinnamaldehyde on biofilm formation and sarA expression by methicillin-resistant Staphylococcus aureus. Lett Appl Microbiol, 2011; 53:409-16.

Khan SN, Khan S, Iqbal J, Khan R, Khan AU. Enhanced killing and antibiofilm activity of encapsulated cinnamaldehyde against Candida albicans. Front Microbiol, 2017; 8:1-15.

Kim YG, Lee JH, Gwon G, Kim SI, Park JG, Lee J. Essential oils and eugenols inhibit biofilm formation and the virulence of Escherichia coli O157:H7. Sci Rep, 2016; 6:1-11.

Longo F, Vuotto C, Donelli G. Biofilm formation in Acinetobacter baumannii. New Microbiol, 2014; 37:119-27. 
Mansour MK, Rhman SAA. Biofilm formation and its association with multiple drug resistance among clinical isolates of Acinetobacter baumanii. Egypt J Med Microbiol, 2012; 21:81-9.

Mohamed SH, Mohamed MSM, Khalil MS, Azmy M, Mabrouk MI. Combination of essential oil and ciprofloxacin to inhibit/eradicate biofilms in multidrug-resistant Klebsiella pneumoniae. J Appl Microbiol, 2018a; 125:84-95.

Mohamed SH, Mohamed MSM, Khalil MS, Mohamed WS. Antibiofilm activity of papain enzyme against pathogenic Klebsiella pneumoniae. J Appl Pharm Sci, 2018b; 8:163-8.

Niu C, Afre S, Gilbert ES. Subinhibitory concentrations of cinnamaldehyde interfere with quorum sensing. Lett Appl Microbiol, 2006; 43:489-94.

Nowotarska S, Nowotarski K, Grant IR, Elliott CT, Friedman M, Situ C. Mechanisms of antimicrobial action of cinnamon and oregano oils, cinnamaldehyde, carvacrol, 2,5-Dihydroxybenzaldehyde, and 2-Hydroxy-5-Methoxybenzaldehyde against Mycobacterium avium subsp. paratuberculosis (Map). Foods, 2017; 6:72.

Ramanathan S, Suda KJ, Fitzpatrick MA, Poggensee L, LaVela SL, Burns SP, Evans CT. Multidrug-resistant Acinetobacter: risk factors and outcomes in veterans with spinal cord injuries and disorders. Am J Infect Control, 2017; 45:1183-9.

Reena AAA, Subramaniyan A, Kanungo R. Biofilm formation as a virulence factor of Acinetobacter baumannii: an emerging pathogen in critical care units A. J Curr Res Sci Med, 2017: 3:74-8.
Ryu SY, Baek W-K, Kim HA. Association of biofilm production with colonization among clinical isolates of Acinetobacter baumannii. Korean J Intern Med, 2017; 32:345-51.

Song JY, Cheong HJ, Noh JY, Kim WJ. In vitro comparison of anti-biofilm effects against carbapenem-resistant Acinetobacter baumannii: imipenem, colistin, tigecycline, rifampicin and combinations. Infect Chemother, 2015; 47:27-32.

Thakre AD, Mulange SV, Kodgire SS, Zore GB, Mohan Karuppayil S. Effects of cinnamaldehyde, ocimene, camphene, curcumin and farnesene on Candida albicans. Adv Microbiol, 2016; 06:627-43.

Thummeepak R, Kongthai P, Leungtongkam U, Sitthisak S. Distribution of virulence genes involved in biofilm formation in multi-drug resistant Acinetobacter Baumannii clinical isolates. Int Microbiol, 2016; 19:121-9.

How to cite this article:

Mohamed SH, Salem D, Azmy M, Fam NS. Antibacterial and antibiofilm activity of cinnamaldehyde against carbapenemresistant Acinetobacter baumannii in Egypt: In vitro study. J App Pharm Sci, 2018; 8(11): 151-156. 